\title{
UN MÉTODO PARA EL SEXAJE POR ADN DE ALPACA AMPLIFICANDO EL GEN SRY MEDIANTE PCR ${ }^{1}$
}

\author{
A Method for SeXing Alpaca DNA by PCR Amplification of the SRY \\ GENE
}

Nino Arias C. ${ }^{2,3}$ y Wilfredo Huanca L. ${ }^{2,4}$

\section{Resumien}

Se desarrolló un método para el sexaje de muestras de ADN de alpacas basado en la amplificación del gen SRY (Sex-determining Region Y chromosome). Se diseñaron nuevos oligonucleótidos primers a partir de la secuencia parcial del gen SRY del guanaco (Lama guanicoe). Luego de la electroforesis, la muestra de alpaca macho mostró una banda SRY de 146 pares base, la cual no aparece en la muestra de alpaca hembra. Luego de la optimización, el procedimiento de PCR para el diagnóstico de sexo fue aplicado a muestras de ADN de 10 alpacas. El sexo (genotípico) diagnosticado mediante el PCR correspondió con el sexo anatómico (fenotípico) en todos los casos. Este estudio demuestra que el presente método se puede aplicar al sexaje de muestras de ADN de alpaca mediante la amplificación del gen SRY usando PCR.

Palabras clave: bioinformática, diagnóstico molecular, PCR, SRY, cromosoma Y, alpaca

\section{AbSTRACT}

A method for determining the sex of DNA samples from alpacas has been developed using amplification of the SRY (Sex-determining Region Y chromosome) gene. New oligonucleotide primers were designed according to the SRY gene partial sequence from guanaco (Lama guanicoe). After electrophoresis, a normal male alpaca showed a single SRY band of $146 \mathrm{bp}$ that did not appear in female alpaca samples. After optimization, the PCR procedure for determination of sex was applied to $10 \mathrm{DNA}$ alpaca samples. The sex (genotypic) as diagnosed by PCR corresponded with anatomical sex (phenotypic) in all cases. This study showed that the present method can be applied for sexing DNA samples from alpacas by PCR amplification of the SRY gene.

Key words: bioinformatics, molecular diagnosis, PCR, SRY, Y chromosome, alpaca

\footnotetext{
${ }^{1}$ Trabajo presentado en el WBC/ICAR 2008 Satellite Meeting on Camelid Reproduction - Budapest, Hungary.

${ }^{2}$ Laboratorio de Reproducción Animal, Facultad de Medicina Veterinaria, Universidad Nacional Mayor de San Marcos, Lima

${ }^{3}$ Dirección actual: Department of Biomedical Sciences / Biochemistry, University of Veterinary Medicine,Vienna,Austria.E-mail: Nino.Arias@vu-wien.ac.at

${ }^{4}$ E-mail: wilfredo.huanca@gmail.com
} 
El logro de crías hembras es deseable especialmente cuando el criador busca incrementar la población animal de su hato, en tanto que embriones de alpaca macho de rebaños élite serían muy deseables para mejorar la calidad genética de la población a través de la inseminación artificial. Actualmente, existen diversas tecnologías que permiten diagnosticar y controlar el sexo de las crías, como es el uso de semen sexado en la inseminación artificial y la transferencia de embriones sexados (Seidel, 2003).

Se han desarrollado metodologías para la identificación del sexo, amplificando segmentos genómicos ubicados en los cromosomas sexuales del animal a través de la técnica de la Reacción en Cadena de la Polimerasa (PCR). Así, la región ZF (Zinc Finger) tiene secuencias homólogas en ambos cromosomas sexuales, siendo utilizada para el sexaje de muestras de ADN de humano, bovino, ovino y caprino (Aasen y Medrano, 1990), búfalo de agua (Pande y Totey, 1998), perros (Murakami et al., 2001), elefante asiático (Vidya et al., 2003), zorro (Ortega et al., 2004), bovinos, porcinos y equinos (Poloumienko, 2004) y de cetáceos (Morin et al., 2005). Del mismo modo, la región SRY (Sex-determining Region Y chromosome), presente únicamente en el cromosoma $Y$, ha sido utilizado para el sexaje de muestras de ADN de llama (Drew et al., 1999), venado (Takahashi et al., 1998) y castores (Kühn et al., 2002). Así mismo, se ha empleado en forma exitosa la amplificación simultánea de SRY y ZF para la determinación del sexo del hombre y de varias especies animales (Pomp et al., 1995).

El presente estudio tuvo por objetivo el desarrollo de una técnica molecular, basada en el PCR, para el sexaje de muestras de ADN de alpaca.

\section{Extracción de ADN Genómico}

Se obtuvo una muestra de $0.5 \mathrm{ml} \mathrm{de}$ sangre en tubos con EDTA de cada una de las 10 alpacas Huacaya (Vicugna pacos) de un mes de edad ( 5 machos y 5 hembras). Se procesaron $0.3 \mathrm{ml}$ de sangre de cada animal conforme las instrucciones del Manual Técnico TM050 (Promega Corporation, 2005) y se empleó el kit comercial de purificación de ADN genómico Wizard $\AA$. El producto fue conservado a $4{ }^{\circ} \mathrm{C}$ hasta su procesamiento.

\section{Diseño y Selección de Primers}

Se utilizó herramientas bioinformáticas disponibles en Internet para diseñar un nuevo par de primers para amplificar el gen SRY de alpaca. Para esto, se utilizó una secuencia parcial de 173 pares base del gen SRY del guanaco disponible con el código de ingreso Nucleotide U66068 en el GenBank del National Center for Biotechnology Information (http://www.ncbi.nlm.nih.gov/). La secuencia U66068 fue ingresada al Servidor Bioinformático de la Universidad de Bielefeld de Alemania (http://bibiserv. techfak.uni-bielefeld.de/) para usar la herramienta GeneFisher (Giegerich et al., 1996). Adicionalmente, se eligió el par de primers publicados por Aasen y Medrano (1999) P15EZ 5' ATAATC ACA TGGAGA GCC ACA AGC T 3' y P2-3EZ 5' GCA CTT CTT TGG TAT CTG AGA AAG T 3' para la amplificación de ZF, el cual ya fue evaluado previamente en llamas (Lama glama) (Pomp et al., 1995).

\section{Protocolo PCR}

Se utilizó tubos para PCR de $0.2 \mathrm{ml} \mathrm{y}$ se preparó un volumen de $100 \mu 1$ de mezcla de insumos (master mix) para cada reacción PCR. Los insumos fueron los siguientes: 10 $\mu 1$ de PCR buffer [10X/ $\mu 1], 10 \mu 1$ de $\mathrm{MgCl} 2$ [25 mM/ $/ \mu \mathrm{l}], 2 \mu \mathrm{l}$ de dNTPs mix $[10 \mathrm{mM} / \mu \mathrm{l}]$, $2 \mu 1$ de primer forward [ $50 \mathrm{pM} / \mu 1], 2 \mu 1$ de pri- 
mer reverse [ $50 \mathrm{pM} / \mu 1], 65.2 \mu 1$ de agua PCR miliQ, $0.8 \mu 1$ de Taq DNA polimerasa [ $5 \mathrm{U} / \mu \mathrm{l}]$ y $8 \mu 1$ de ADN genómico de cada animal [ $\sim 50$ $\mathrm{ng} / \mu \mathrm{l}]$.

Se colocó los tubos en un ciclador térmico PTC-100 Engine ${ }^{\mathrm{TM}}$ (MJ Research Inc., USA) y las muestras fueron desnaturalizadas (separación de hebras) a $94{ }^{\circ} \mathrm{C}$ por $5 \mathrm{~min}$, seguidos de 35 ciclos a las siguientes temperaturas: desnaturalización de hebras a $94{ }^{\circ} \mathrm{C}$ por $45 \mathrm{~s}$, hibridación de primers a las hebras a $58^{\circ} \mathrm{C}$ por $45 \mathrm{~s}$ y extensión de los primers por la polimerasa a $72^{\circ} \mathrm{C}$ por $1 \mathrm{~min}$. Finalmente, se aseguró la extensión a $72{ }^{\circ} \mathrm{C}$ por $10 \mathrm{~min}$.

Se analizó los productos PCR (amplicones) mediante electroforesis en un gel de agarosa al $2 \%$ teñida con bromuro de etidio, $\mathrm{y}$ visualizada bajo luz ultravioleta. Se fotografió los geles usando el sistema UVP de trasluminador ultravioleta y cámara fotográfica digital (Bio Doc-it and Visi Doc-it Systems Ultra-Violet Products Ltd., Cambridge UK).

\section{Secuenciamiento}

Con la finalidad de conocer el número y la secuencia de nucleótidos, se purificó el producto amplificado por el PCR del gen SRY de una alpaca macho en un secuenciador ABI PRISM® 310 Genetic Analyzer (Applied Biosystems). La homología de la nueva secuencia lograda de la alpaca con la del guanaco se analizó con la herramienta bioinformática Clustal W del Instituto Europeo de Bioinformática (http://www.ebi.ac.uk/ clustalw/).

\section{Resultados}

Siguiendo los parámetros que presentó por defecto GeneFisher, se logró la nueva secuencia de oligonucleótidos primer: 5'-3 CAT TGT ATG GGC TCG TGA (forward) y TTC GAG GAG GCA CAG AG (reverse), que amplificó un producto comprendido entre las bases 23 al 150 de la secuencia U66068.
Mediante la interpretación de la primera electroforesis, se verificó la presencia de una banda común en la hembra y el macho (correspondientes a ZF) y de una banda exclusiva en el macho (gen SRY).

El método fue evaluado en 10 muestras de ADN de alpacas con sexo fenotípico conocido. En la segunda electroforesis se observa una banda exclusiva de las muestras correspondientes a alpaca macho. Todas las determinaciones de sexo usando PCR fueron semejantes al sexo fenotípico de las alpacas muestreadas.

El secuenciamiento del producto PCR del gen SRY de alpaca identificó una secuencia de 146 pares bases con $100 \%$ de homología con la secuencia parcial del gen SRY de guanaco (Nucleotide U660668). Esta nueva secuencia fue enviada para su registro en el GenBank en fecha 20 de julio de 2006 y publicada online el 29 de agosto de 2006 con el código de ingreso Nucleotide DQ862123 y la definición "Lama pacos sexdetermining region Y gene, partial cds". Adicionalmente, se publicó la secuencia de sus 48 aminoácidos con el código de ingreso Protein ABI31754 y la definición "sexdetermining region Y [Lama pacos]".

\section{Discusión}

A partir del sexaje de muestras de ADN de llamas realizado a través de la amplificación del gen ZF (Pomp et al., 1995) y la amplificación del gen SRY (Drew et al., 1999) se pudo adaptar la técnica PCR para su uso en alpacas.

La amplificacion del gen SRY demostró indirectamente la presencia del cromosoma $\mathrm{Y}$ en las muestras de ADN de alpacas macho, de manera similar al hallazgo de Drew et al. (1999) en llamas. No obstante, el cromosoma Y puede ser identificado directamente mediante la técnica citológica del cariotipo de acuerdo a su for- 
ma y tamaño, y este cariotipo en camélidos sudamericanos es conocido desde el primer registro de Cappana et al. (1965) hasta el reciente de Zapata (1999).

La amplificación del gen ZF en diversas especies animales se debe a que es una región que aparentemente se ha mantenido conservada a lo largo de la evolución (sin demasiados cambios en las bases de nucleótidos que lo conforman). Este enunciado ha sido sugerido luego del análisis comparativo de los genes ZFX y ZFY bovino, porcino y equino realizada por Poloumienko (2004) y la amplificación del gen ZF en humanos y diversas especies de animales domésticos y silvestres realizados por Pomp et al. (1995). Estos hallazgos sugieren que puede haber ocurrido la misma conservación evolutiva en los camélidos sudamericanos, por lo que se justifica el uso de los primers desarrollados por Aasen y Medrano (1990) para amplificar la región ZF (conformado por ZFY asociado al cromosoma Y y ZFX asociado al cromosoma X).

El uso de herramientas bioinformáticas, logradas principalmente a través del Proyecto Genoma Humano (Baxevanis, 2001), permitió encontrar mediante el servidor NCBINational Center of Biotechnology Information, EEUU, una secuencia del gen SRY de guanaco (NCBI Nucleotide U66068) y el reporte de su aplicación en el sexaje satisfactorio de ADN de la llama (NCBI PubMed 10530329) (Drew et al., 1999). La misma información puede obtenerse usando los servidores EMBL-European Molecular Biology Laboratory de Alemania (http://www.emblheidelberg.de/) y DDBJ-DNA Data Base of Japan (http://www.ddbj.nig.ac.jp/). Así mismo, el uso de GeneFisher del Servidor Bioinformático de la Universidad de Bielefeld, Alemania (http://bibiserv.techfak.unibielefeld.de/) permitió el diseño interactivo de primers ingresando la secuencia Nucleotide U66068. También se utilizó la herramienta Clustal W del Instituto Europeo de Bioinformática (http://www.ebi.ac.uk/ clustalw/) para el alineamiento de las secuen- cias logradas por el secuenciamiento. Estas herramientas y otras similares permiten el desarrollo acelerado de estudios genómicos (Meyers-Wallen, 2006).

Los resultados del presente estudio demuestran la factibilidad de identificar el sexo de la alpaca a partir de muestras de sangre y sugieren la factibilidad de la identificación del sexo en muestras de semen o embriones preimplantacionales. El logro de crías de alpaca, con el sexo deseado, aprovechando las características productivas favorables de determinado sexo, permitirá contribuir con un valor agregado a las tecnologías de inseminación artificial y transferencia de embriones en alpacas.

\section{Agradecimientos}

Los autores expresan su agradecimiento al Dr. Armando Hung por brindar el acceso a las facilidades de la Universidad Peruana Cayetano Heredia, Lima; y al Dr. Massoud Malek del Organismo Internacional de Energía Atómica, Viena, por su comentarios y presentación del trabajo en el encuentro de Budapest. Asimismo, se agradece la beca de investigación otorgada por el CONCYTEC PROCOM.

\section{Conclusiones}

Se desarrolló una técnica de PCR para identificar el sexo a partir de muestras de ADN genómico de alpaca obtenidas de sangre, amplificando parcialmente al gen SRY de Alpaca (Nucleotide DQ862123).

\section{Literatura Citada}

1. Aasen E, Medrano J. 1990. Amplification of the ZFY and ZFX genes for sex identification in humans, cattle, sheep and goats. Nat Biotechnol 8: 12791281. 
2. Baxevanis A. 2001. Bioinformatics and the internet. In: Bioinformatics, a practical guide to the analysis of genes and proteins. Cap. 1. New York: Ed. John Wiley \& Sons. p 1-17.

3. Cappana E, Mangili G, Civitelli MV, Conti L, Dotti E. 1965. Prime osservazioni sulla Cariologia dei Camelidi. Boll Zool 31: 71-77.

4. Drew M, Meyers-Wallen V, Acland G, Guyer C, Steinheimer D. 1999. Presumptive Sry-negative XX sex reversal in a llama with multiple congenital anomalies. J Am Vet Med Assoc 215: 1134-1139.

5. Giegerich $R$, Meyer F, Schleiermacher C. 1996. GeneFisher - Software support for the detection of postulated genes. Proc IV International Conference on Intelligent Systems for Molecular Biology 4: 68-77.

6. Kühn R, Schwab G, Schröder W, Rottmann O. 2002. Molecular sex diagnosis in castoridae. Zoo Biol 21:305-308.

7. Meyers-Wallen V. 2006. Genetics, genomics, and molecular biology of sex determination in small animals. Theriogenology 66: 1655-1658.

8. Morin PA, Nestler A, Rubio-Cisneros NT, Robertson KM, Mesnick SL. 2005. Interfamilial characterization of a region of the ZFX and ZFY genes facilitates sex determination in cetaceans and other mammals. Mol Ecol 14: 3275-3286.

9. Murakami M, Fujise H, Lee YS, Matsuba C, Fujitani H. 2001. Reliable sex identification of dogs by modified PCR/PFLP analysis. J Vet Med Sci 63: 679-681.

10. Ortega J, Franco MR, Adams B, Ralls K, Maldonado J. 2004. A reliable, noninvasive method for sex determination in the endangered San Joaquin kit fox
(Vulpes macrotis mutica) and other canids. Conserv Genet 5: 715-718.

11. Pande A, Totey SM. 1998. ZFX and ZFY loci in water búfalo (Bubalus bubalis): Potential for sex identification. Genet Anal Biomol E 14: 85-88.

12. Poloumienko A. 2004. Cloning and comparative analysis of the bovine, porcine, and equine sex chromosome genes ZFX and ZFY. Genome 47: 74-83.

13. Pomp D, Good BA, Geisert RD, Corbin CJ, Conley AJ. 1995. Sex identification in mammals with polymerase chain reaction and its use to examine sex effects on diameter of day 10 or 11 pig embryos. J Anim Sci 73: 1408-1415.

14. Promega Corporation. 2005. Isolating genomic DNA from whole blood. In: Wizard $\AA$ genomic DNA purification kit. Technical Manual TM050. Madison, WI. USA.

15. Seidel G 2003. Economics of selecting for sex: the most important genetic trait. Theriogenology 59: 585-598.

16. Takahashi M, Masuda R, Uno H, Yokoyama M, Susuki M, Yoshida M, Ohtaishi N. 1998. Sexing of carcass remains of the Sika Deer (Cervus nippon) using PCR amplification of the SRY gene. J Vet Med Sci 60: 713-716.

17. Vidya T, Kumar R, Arivazhagan C, Sukumar R. 2003. Application of molecular sexing to free-ranging Asian elephant (Elaphas maximus) populations in southern India. Curr Sci India 85: 10741077.

18. Zapata B. 1999. Diferenciación de camélidos sudamericanos mediante el análisis de cariotipo. Tesis de Magíster. Santiago de Chile: Pontificia Universidad Católica de Chile. 140 p. 